\title{
A comparison of the effects of formal similarity among trigrams and among word triads*
}

\author{
BENTON J. UNDERWOOD and JOEL ZIMMERMAN \\ Northwestern University, Evanston, Illinois 60201
}

\begin{abstract}
The central question of this report concerned the role of formal similarity in free recall of lists of trigrams and lists of three-letter word triads. Similarity was manipulated among trigrams by duplicating letters and among triads by duplicating words. An initial study showed that lists of 16 letters were learned more rapidly than a list of 16 three-letter words. Therefore, in the major experiment, the Ss were given all appropriate elements on test trials so that only associative learning was required. Increases in formal similarity caused decreases in rate of learning for both types of lists, and the mechanisms of the interference seemed to be the same for both types of lists. However, the learning of the trigram lists was more rapid than the learning of the triad lists, the difference being maximal with low similarity.
\end{abstract}

Although formal similarity of verbal units may be defined by rating procedures (Runquist \& Joinson, 1968), it is more commonly specified in terms of the number of different elements involved in constructing a list. If the list consists of six trigrams, the use of 12 different letters to construct the trigrams results in higher formal similarity than the use of 18 different letters. No attempt will be made here to review the vast literature on the relationships between formal similarity and learning. It is sufficient to say that, at least for items that are not words, the decrease in learning performance as formal similarity increases is large, and this relationship holds for all of the commonly used laboratory learning tasks. The problems of interpretation, with special emphasis on formal similarity among the stimulus terms in a paired-associate list, have been examined by Runquist (1972). His discussion was based upon the letter as the basic unit of interference, since this has been the unit most frequently used to manipulate formal similarity. In the present report, the three-letter word was used as the unit to manipulate formal similarity and a comparison was made with the letter as the unit in trigrams.

The most profound effects of similarity emerge from studies in which low-meaningful consonant trigrams, such as GKW, have been used. Such an item is very difficult to pronounce and is likely to be processed by a spelling response, G-K-W. Suppose, therefore, a word triad is used that appears to have the same processing properties, a triad such as GUN-KEY-WEB. This item, it would seem, must be processed as three distinct elements, just as in the case of GKW. To pronounce the three words in order might require slightly more time than to spell GKW, but the difference would certainly not be great.

*This research was supported by the Personnel and Training Research Programs, Psychological Sciences Division, Office of Naval Research under Contract N00014-67-A-0356-0010, Contract Authority Identification NR 154-321. Reproduction in whole or in part is permitted for any purpose of the United States Government.
Assume that a free-recall list is constructed of trigrams and another list of an equal number of word triads, with no repetition of letters among the trigrams and no repetition of the words among the triads. Which list would be learned most rapidly? In terms of the analysis given above, the number of elementary units to be processed is the same in both cases. If such processing is critical for learning, no difference should be anticipated in learning the trigrams and the triads. Such equivalence seems to have been supported by Murdock (1961) in his demonstration that the short-term forgetting of trigrams and word triads did not differ. In a study of stimulus selection, Berry and Cole (1973) reached the conclusion that the processing of trigrams and triads was very similar and that they may be considered equivalent research models. Therefore, based upon a process analysis and upon past work, it would seem that the free-recall learning of trigrams and triads should be equivalent

The above expectation not withstanding, it would appear that trigrams and word triads would differ in the likelihood that semantic or meaning responses would be evoked spontaneously. These responses should be relatively frequent for the triads as compared to the trigrams. Insofar as such semantic responses enter positively into associative learning, it would seem that a prediction should favor the triads. In a more formal sense, the perceptual or processing responses (the representational responses) may be roughly equivalent for the triads and trigrams, but the possibilities of elaborative or semantic responses would appear to be greater for the triads.

Next, consider the influence of formal similarity. In one case the number of repeated letters is increased among the trigrams in the list and in the other the number of repeated words is increased. In both cases the expectation would be an increase in learning difficulty due to the repeated representational responses (see Runquist, 1972, for possible mechanisms). In addition, for the word triads there could be further interference from the semantic responses. That is, if identical 


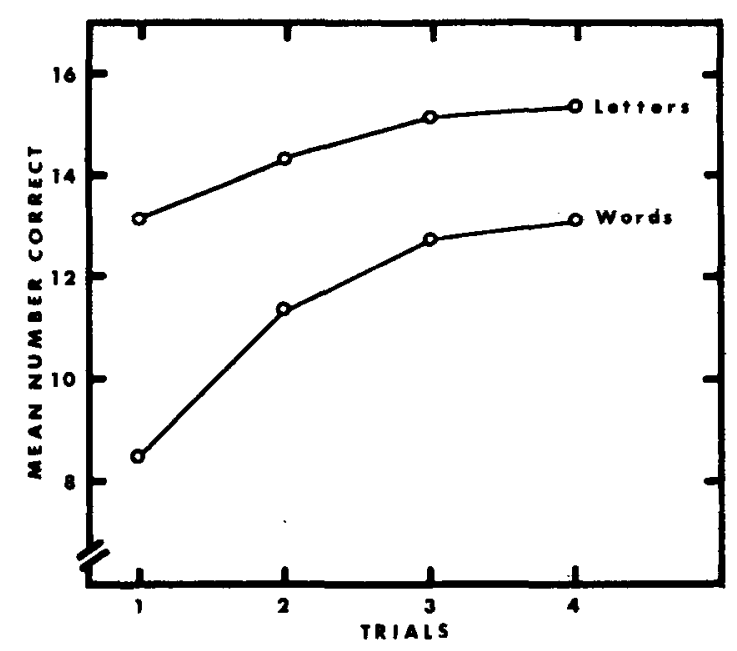

Fig. 1. Free-recall learning of 16 letters and 16 three-letter words.

representational responses result in interference, it would seem reasonable to expect that identical semantic responses would also produce interference. If this occurs, it leads to the prediction that increases in formal similarity would result in a greater decrement in the learning of word triads than in the learning of trigrams. Let it be clear, however, that this study was undertaken with the primary purpose being simply that of comparing the effects of repeated elements in trigrams and triads with the belief that any differences which emerged would be attributed to the differences in semantic responses evoked by the two different kinds of materials.

The original intent was to use free-recall learning. However, the interest was in associative learning of the elements (letters and words) within each item. The learning of the elements per se might well differ for free recall, and certainly such learning will differ as formal similarity increases for both kinds of elements. The first experiment simply demonstrated that the free-recall of letters and words does differ. Therefore, in the second and major experiment, all elements were provided the $S$ so that learning consisted only of acquiring the ordering of the elements within each item.

\section{EXPERIMENT I}

\section{Method}

\section{Lists}

There were two lists of 16 items each. The letter list was made up of the 16 lowest frequency letters in the alphabet: b, c, d, f, $\mathrm{g}, \mathrm{j}, \mathrm{k}, \mathrm{m}, \mathrm{p}, \mathrm{q}, \mathrm{u}, \mathrm{v}, \mathrm{w}, \mathrm{x}, \mathrm{y}, \mathrm{z}$. The 16-letter words had Thorndike-Lorge (1944) frequencies of from one to six: yew, tab, par, wry, gym, que, fad, keg, imp, dun, ban, jot, rig, lax, vie, $n o b$. Four random orders of the letters were determined and, with a word yoked alphabetically to a letter, the same four random orders were used for the words.

\section{Procedure and Subjects}

Four alternating study and test trials were given. Each word or letter was exposed on a memory drum for $3 \mathrm{sec}$ each on the study trials, and $60 \mathrm{sec}$ were allowed for free recall. The instructions indicated that the order of recall was unimportant. Strong instructions were given against guessing.

There were 30 Ss. Each S learned both lists, 15 having four study-test trials on the letter list followed by the four trials on the word list and $15 \mathrm{Ss}$ having the reverse order.

\section{Results}

The mean number correct on each trial for the two lists is shown in Fig. 1. It is quite obvious that the list of letters was acquired more rapidly than the list of words. Performance on the first trial of the letter list was identical to that on the fourth trial for the word list. The statistical analysis included trials and order in which the two lists were learned. The only significant sources of variance other than trials was type of list $[F(1,28)=87.62, p<.01]$ and the Type of List by Trials interaction $[F(3,84)=11.94, p<.01]$. This interaction is attributed to the near asymptotic performance on the letter list for the third and fourth trials.

It is, perhaps, no surprise that the letter list was learned more rapidly than the word list. The acquisition of the letter list may become a recognition task in the sense that the $S$ need only select the 16 correct letters from among the 26 possible. This would likely lead to alphabetical organization in recall. Using a technique suggested by Thompson (1973), the alphabetical organization was determined for both lists for the firs 1 and fourth trials. The technique yields values ranging from 0 (no organization) to 1 (perfect organization) The mean values for the letters were .26 and .58 for Trials 1 and 4 , respectively. For the word list, the corresponding values were .09 and .12 . Statistically, the main effects of type of material $(F=44.41)$ and trials $(F=30.38)$ were reliable, as was also their interactior $(F=17.12)$, with all $F s$ based on $\mathrm{df}=1 / 28, \mathrm{p}<.01$.

Whether the entire difference between words anc letters can be accounted for by an alphabetica: recognition strategy for letters is a moot issue. The degree of alphabetical organization on the first trial fol the letters was not great, but the Ss may not have beer systematic in their search initially. Analysis of first-tria recall as a function of serial position of occurrence or the study trial showed comparable primacy and recency effects for both lists, suggesting that factors in additior to search strategy influenced performance. Thr differences between the performances on the two list: cannot be attributed to guessing differences. It will be remembered that the $S$ had been instructed not to guess Across all Ss on all trials there was a total of 17 lette: intrusions and 20 word intrusions. Thus, although the data indicate that the alphabetical recognition strateg) was probably important in the better performance or 
the letter list than on the word list, there is no way of knowing whether or not it is responsible for all of the observed differences.

The results leave no doubt that a list of 16 letters is acquired more rapidly than a list of 16 three-letter words. It appeared, therefore, that to study the comparative associative learning of trigrams and word triads, element recall should be eliminated from the task.

\section{EXPERIMENT II}

The purpose of Experiment II was to study the effects on learning of formal similarity among letters in trigrams and among words in word triads.

\section{Method}

\section{Materials}

Eight lists were used, each consisting of eight items. Four of these were trigram lists and four were word-triad lists. The method of constructing the lists, shown in Table 1, will be described in detail. The zero-similarity trigram list was devised first. This list consisted of 24 different letters (a and e were not used) and each trigram in the list was constructed so as to have minimal interletter association values, as determined from the Underwood and Schulz (1960) tables. None of the trigrams in any of the lists occurs as a three-letter sequence in words according to Appendix D in Underwood and Schulz. The zero-similarity list will be designated List T-0. For the first level of similarity, four letters were repeated (List T-4). A repetition always occurred across trigrams, and the same letter never occupied the same position in two trigrams. In List T-8, eight letters were repeated and, in List T-12, 12 letters. Since no letter occurred more than twice in a list, List T-12 contained 12 letters, each occurring twice.

In order to construct List $\mathrm{T}-4$, then $\mathrm{T}-8$, and finally $\mathrm{T}-12$, it was necessary to drop out the high-frequency letters in order to keep the interletter association values constant across the four lists. The letters $i, n, o$, and $t$, used in List $T-0$. were used in no other list. For List T-8, the letters $h, 1, r, s$, used in $T-4$, were dropped and the remaining 16 letters were used in constructing List T-8. Finally, for List T-12, c, d, m, and $u$ were discarded and the trigrams were constructed from the remaining 12 letters. As a consequence, the summed interletter associations for the four lists in order were $31,28,26$, and 32 . These sums consist of the values between the first letter and the second and between the first two letters and the third.

The first step in constructing the four word-triad lists (W-0, W-4, W-8, W-12) was to choose 24 three-letter words, each beginning with a different letter, among which the repetition of all letters was relatively low. It was not possible, of course, to avoid some letter repetition, but as may be seen in Table 1, all possible types of consonant and vowel combinations were used, this being done particularly to minimize repetition of middle letters. These words varied in frequencies between 1 and 13 in Thorndike and Lorge (1944). It will be noted that there is some acoustic similarity among the words, e.g., hue, yew, mew, a deliberate inclusion to parallel grossly the acoustic similarity that exists among letters.

The 24 letters were yoked with the 24 words in terms of their frequencies. Thus, ale $(\mathrm{f}=13)$ was yoked with $\mathrm{i}$, hue $(\mathrm{f}=12)$ with $t$, and so on. In forming the triads for List $W 4$, the four words omitted (which had occurred in List W-0) were those yoked with the four high-frequency letters which, it will be
Table 1

Trigram Lists (T-0 Through T-12) of Increasing Similarity and Word-Triad Lists of Comparable Similarity (W-0 Through W-12)

\begin{tabular}{cccc}
\hline T-0 & T-4 & T-8 & T-12 \\
\hline cfy & cfy & cfy & bfv \\
igw & lgw & qgw & kqj \\
khj & khq & kwq & ygw \\
dsu & dsu & dku & xkf \\
xbn & xbv & xbv & pvz \\
ozq & pvj & pvj & wpx \\
tpm & ypm & ypc & jzg \\
rvl & rzl & jzm & qby \\
\hline W-0 & W-4 & W-8 & W-12 \\
\hline yew-dun-wry & yew-dun-wry & yew-dun-wry & ban-dun-nob \\
ale-fad-tab & ode-fad-tab & lax-fad-tab & jot-lax-gym \\
jot-mew-gym & jet-mew-lax & jot-tab-lax & wry-fad-tab \\
imp-urn-par & imp-urn-par & imp-jot-par & vie-jot-dun \\
vie-ban-cod & vie-ban-nob & vie-ban-nob & keg-nob-que \\
elf-que-lax & keg-nob-gym & keg-not-gym & tab-keg-vie \\
hue-keg-rig & wry-keg-rig & wry-keg-yew & gym-que-fad \\
sip-nob-ode & sip-que-ode & gym-que-rig & lax-ban-wry \\
\hline
\end{tabular}

remembered, were dropped when constructing List $\mathrm{T}-4$. The yoking of letters and words also allowed the positions of the repetitions of letters and words to be exactly the same. For example, in List $\mathbf{T}-4$, the letter 1 occurs in the first position of the second trigram and in the third position of the eighth trigram. It can be seen that in List W-4 the word ode, yoked with 1 , also occurs in the first position of the second triad and in the third position of the eighth. Across the lists, positions of repetitions were exactly the same for the trigram and for the word lists.

\section{Procedure and Subjects}

There were eight study and test trials given on each list. The letters for both types of lists were printed in lowercase. The letters in the trigrams were separated by a space, as were the words in the triads. Each item was presented on a memory drum for $3 \mathrm{sec}$ for study. Immediately after the presentation of the last item, $\mathrm{S}$ was given a sheet on which he recorded his responses. At the top of this sheet the letters appropriate for the trigrams or the words appropriate for the triads were listed alphabetically. Thus, for List T-0 there were 24 letters listed, for T-12 there were 12 letters. Correspondingly, for W-0 there were 24 words, for W-12 12 words. The Ss were instructed to write in the blanks provided all of the correct trigrams or correct triads they could. They were further instructed that the order in which they wrote the items was unimportant but that to be counted correct the three elements within each item must be in the order shown on the study trial. A $90-\mathrm{sec}$ period was allowed for writing the trigrams or triads after each study trial.

Each list was learned by a different group of Ss, there being $24 \mathrm{Ss}$ in each of the eight groups. The list assigned to a particular $\mathrm{S}$ was determined by a block randomized schedule of the eight lists.

\section{Results}

The acquisition curves are shown in the four panels of Fig. 2. The most apparent finding from these data is that the trigram lists are learned more rapidly than the triad lists. This is true at all levels of similarity on all trials. 


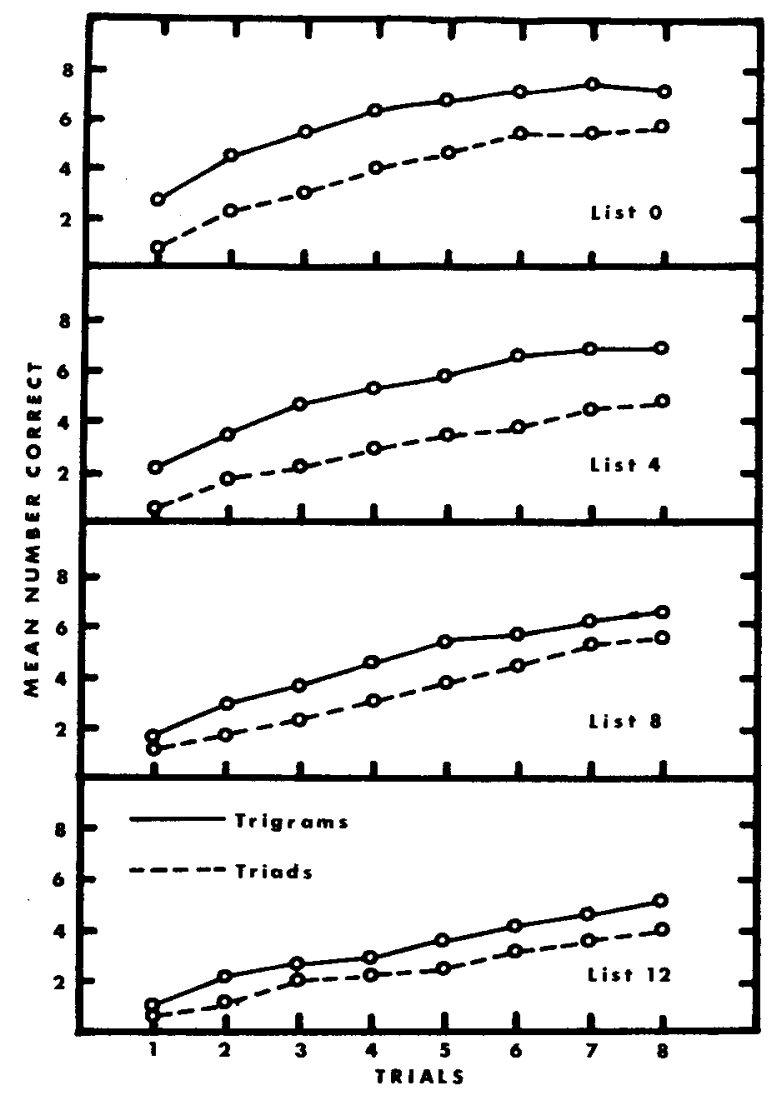

Fig. 2. Associative learning for trigrams and word triads as a function of formal similarity. List $\mathbf{0}$ represents zero similarity, List 12 high similarity.

The differences appear larger with the lower levels of similarity (Lists 0 and 4 ) than with the higher levels (Lists 8 and 12). Looking down the panels shows that, as formal similarity increased, performance decreased.

The results are condensed in Fig. 3, in which is shown the mean number of correct responses across all eight trials for all eight lists. Clearly, performance decreased as similarity increased, and the trigrams were easier to learn than the triads. No reason has been discovered as to why List W-8 was learned more rapidly than List W-4. Statistically, the type of list (trigrams vs triads) was a significant source of variance $[\mathrm{F}(1,184)=70.08$, $\mathrm{p}<.01]$, as was similarity $[\mathrm{F}(3,184)=20.52, \mathrm{p}<.01]$. The interaction between the two variables was also reliable $[F(3,184)=2.81, p<.05]$. The meaning of the interaction is somewhat ambiguous because of the nonmonotonicity in the triad curve. However, a slope analysis showed that only the linear component of each curve was significant statistically, and the difference in the linear slopes was reliable $[F(1,184)=6.50, p<.05]$. Actually, the interaction is probably underestimated because of a ceiling effect for List T-0. Nineteen of the 24 Ss had all items correct on the last two trials. To avoid this restriction, an analysis was carried out on the first four trials only and revealed a highly reliable interaction of the linear slopes $[F(1,184)=10.65$, $\mathrm{p}<.01]$. It seems appropriate to conclude that the two variables interact and that, at some level of similarity higher than those used here, the two types of materials might be learned at the same rate, albeit the rate would be very slow. Even in the present experiment, for List W-12 the average $\mathrm{S}$ was getting only four correct responses after eight study trials.

The interaction noted above was not as expected by the analyses given in the introduction. As was pointed out, duplicating words should result in two sources of interference, interference from representational responses and interference from semantic responses. Based upon the assumption that interference from semantic responses would be minimal with the trigrams, it would seem that as formal similarity increased the difference in performance on words and trigrams should increase. Instead, the data showed a decrease in the difference. This problem of interpretation is tied closely to the unexpected finding that with zero similarity (Lists $\mathrm{T}-0$ and $\mathrm{W}-0$ ) the triads proved a far more difficult task than did the trigrams.

The difference that occurred on the first trial was a magnitude of two items. To be sure, the words were of low frequency but, except for two or three of them (que, vie, wry), the meaning should have been apparent to the college student Ss. The summed frequency of the words in each triad did not correlate $(-.17)$ with the learning of the items, although admittedly the frequency range was quite restricted.

A number of further analyses were undertaken to see if any clues could be found to account for the differences in learning across all levels of similarity.

(1) There were strong recency effects in that the last item presented on a study trial was likely to be given on the test trial. These effects did not differ for trigrams and triads, and they did not vary as a function of similarity.

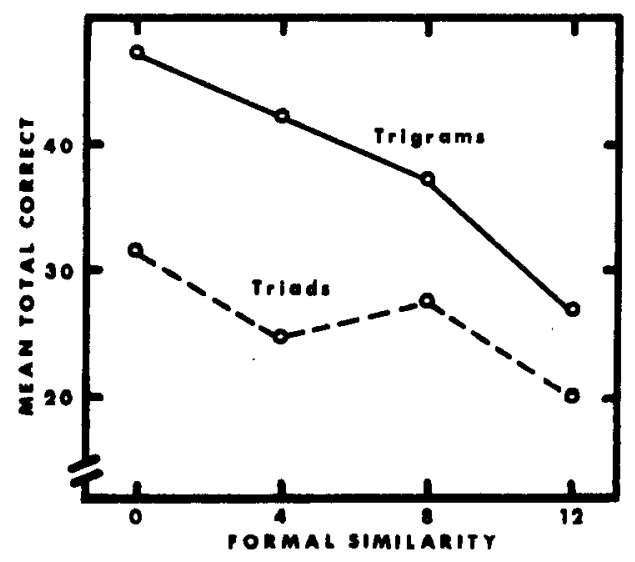

Fig. 3. Total correct responses over eight trials as a function of formal similarity for trigram lists and triad lists. The standard deviations for the word triads were $12.73,8.07,8.80$, and 6.20 in order from left to right; the corresponding values for the trigrams were $9.86,10.24,12.26$, and 10.25 . 
(2) Lists T-0 and W-0 were examined to determine the lature of errors that were made. For this purpose, hree-element responses were examined in which any wo of the elements were correctly placed. The question soncerned the third element that was an incorrect slement for that particular item (as defined by two of he elements being correct). What correct element did his incorrect element replace was the question asked. Since two elements were correct (and $S$ would not epeat an element within an item) and since a particular slement could not replace itself, there were 441 cells in he error matrix. For the letters for all eight trials, there were entries in 57 cells representing 93 erroneous esponses. The maximum number of entries in any cell was five, this occurring for two cells. In one, $\mathrm{c}$ replaced $\mathrm{r}$ as the correct response and, in the other, $\mathrm{c}$ replaced $\mathrm{x}$. There were three cells with four entries, i replacing $g, m$ ceplacing $\mathrm{w}$, and $\mathrm{s}$ replacing $\mathrm{z}$. For cells with three intries, the letters were $b$ for $g, b$ for $p, n$ for $m, v$ for $g$, and $v$ for $i$. An examination of the replacements indicates some evidence for acoustic confusion, but it is oy no means prominent in these data.

The corresponding analysis for List W-0 showed entries in 78 cells and a total of 113 errors. Only three zells had as many as five errors; in the three cells hue replaced yew, lax replaced tab, and sip replaced hue. There were no cells with four entries but five with three intries: ale for elf, ban for dun, ban for fad, elf for jot, and gym for dun. As with the letters, there is some evidence for acoustic confusion.

(3) The correct placement of elements was examined as a function of position (first, second, third) within the trigrams and triads. All correctly placed elements were included regardless of the correctness of the other alements given with it. Incomplete items were also used if an element of the incomplete item was correctly positioned. The first three trials of the four extreme lists (T-0, W-0, T-12, W-12) were examined. The probability of correct placement was equivalent across the three positions for all four lists on all three trials, although, of course, the absolute probabilities differed across levels of similarity and type of material.

(4) In one way of viewing the data, the change in performance as a function of similarity differs but little for the trigrams and triads. Using List $T-0$ as a base, the decrease in performance between this list and List T-12 was $43 \%$; the decrease from List $\mathrm{W}-0$ to List $\mathrm{W}-12$ was $37 \%$. This suggests that increasing similarity was accompanied by quite comparable changes in performance for both types of lists. This possibility will be examined in another manner.

Lists T- $0, \mathrm{~W}-0, \mathrm{~T}-12$, and $\mathrm{W}-12$ may all be considered homogeneous lists. In the first two lists there were no duplicated elements; in the last two all were duplicated. The other lists were heterogeneous in that there were varying amounts of duplication of elements. For example, in Lists $\mathrm{T}-4$ and $\mathrm{W}-4$, two items had no duplicated elements, four had one, and two had two.
Heterogeneous lists should show greater variability in item difficulty than should homogeneous lists, hence the reliability of the item difficulties should be higher. Each group of $24 \mathrm{Ss}$ was divided randomly into two subgroups of 12 each, and the total correct responses for each item over eight trials was determined for each subgroup independently. Product-moment correlations were then calculated between the scores for the subgroups to determine item reliability. For the trigram lists, from List $\mathrm{T}-0$ to $\mathrm{T}-12$, the values were $.59, .81, .79$, and .45 . The corresponding values for the triad lists were $.42, .86$, .73 , and .46 . These data suggest that the interference is item specific for both types of lists. The data for particular items indicated this specificity directly in that those with the fewer duplicated letters were learned more readily than were those with the greater number of duplicated letters in Lists T-4, T-8, W-4, and W-8.

\section{DISCUSSION}

All of the data indicated that formal similarity influenced trigrams and word triads in much the same way. The interference resulting from duplicated elements was quite element specific and increased as the number of duplicated elements increased.

The puzzle of the current findings is why, with zero similarity, the triads were so much more difficult to learn than were the trigrams. Writing time per unit would be greater for triads than for trigrams, but this difference was of no moment on the early trials where few correct responses were produced. The time to read three words may be slightly longer than the time to read three letters, but this time difference should be inconsequential when the study interval was $3 \mathrm{sec}$.

In the introduction it was pointed out that the two types of material should differ in terms of the implicit semantic response that they elicit. If this is an appropriate premise, it follows that a greater number of attributes was contained in the memory for the words than for the letters. If these additional attributes (or the additional elements of information) are not differentially effective for associative learning (if they do not include more effective associative devices than is present in the memory for the letters), then the learning of the triads could be retarded. In effect, the $S$ must sort through information, some of which is not useful. A few Ss voluntarily reported that during learning of the triad list they discovered that no letter was used as the first letter of a word more than once. They then created a single trigram out of the first letters of the three words within a trigram, using this first-letter information to identify the appropriate words on the tests. It is not known how frequently this selection occurred, nor whether learning was facilitated thereby, but such reports do suggest the possibility that the semantic information was not particularly useful and was, perhaps, even detrimental for this associative learning task. Learning as a result of contiguity of 
representational responses may be a relatively efficient form of associative learning in some situations.

\section{REFERENCES}

Berry, F. M., \& Cole, S. R. Stimulus selection in paired-associate learning: Consonant-triad versus word-triad paradigms. Journal of Experimental Psychology, 1973, 97, 402-404.

Murdock, B. B., Jr. The retention of individual items. Journal of Experimental Psychology, 1961, 62, 618-625.

Runquist, W. N. Intralist interference and stimulus similarity. In C. P. Duncan, L. Sechrest, and A. W. Melton (Eds.), Human memory: Festschrift for Benton J. Underwood. New York: Appleton-Century-Crofts, 1972.
Runquist, W. N., \& Joinson, P. A. Rated similarity of low association value CVC trigrams. Journal of Verbal Learning \& Verbal Behavior, 1968, 7, 317-320.

Thompson, W. D. Measurement of alphabetical organization in free recall. Psychological Reports, 1973, 32, 1043-1047.

Thorndike, E. L., \& Lorge, I. The teacher's word book of 30,000 words. New York: Columbia University Press, 1944.

Underwood, B. J., \& Schulz, R. W. Meaningfulness and verbal learning. Philadelphia: Lippincott, 1960.

(Received for publication August 10, 1973; revision received September $17,1973$. 